\title{
One-dimensional flow analysis of steady pressure field around a train running in a long tube with a constant width slit
}

\author{
Masanobu IIDA* and Katsuhiro KIKUCHI* \\ * Railway Technical Research Institute \\ 2-8-38 Hikari-cho, Kokubunji-shi, Tokyo 185-8540, Japan \\ E-mail: iida.masanobu.14@rtri.or.jp
}

Received: 6 January 2021; Revised: 30 April 2021; Accepted: 4 June 2021

\begin{abstract}
A one-dimensional theoretical analysis is made of the pressure field around a train running in a long tube with a constant width slit, which is a simplified model of partially enclosed structures (or vented tubes) of railway, such as snow shelters or whole covered stations. It is shown from field measurement results in Shinkansen that the pressure field produced in such partially enclosed structures exhibits a pattern of 'a combination of single sawtooth and its reverse'. The present analysis of a tube with a slit reproduces this unique pressure pattern and shows that one-dimensional pressure field ahead of the train nose and tail can be expressed by elementary functions. It also reveals that the magnitude of the pressure change around the tail can exceed that around the nose, which is also a unique feature that is different from the cases of open air spaces and fully enclosed spaces (i.e., tunnels).
\end{abstract}

Keywords : Pressure distribution, One-dimensional flow, Pipe flow, Vented tube, High-speed train

\section{Introduction}

On the Shinkansen lines in areas with heavy snowfall, snow shelters covering the entire track and 'whole covered stations', which cover most of the platform floor with a roof and side walls, are often built to prevent snow damage. Furthermore, even in areas with low snowfall, soundproof shelters are sometimes installed to reduce the impact of noise or micro-pressure waves by high-speed trains on the environment of wayside residential areas. These shelters and whole covered stations are usually 100-400 m or more in length and can be regarded as a kind of tunnel structure. It is known that, when a train runs in a high-speed railway tunnel, pressure fluctuations occur in the tunnel. One cause of this is pressure waves which are generated by train entry and subsequently go back and forth in the tunnel multiple times. The other is quasi-steady pressure field around the moving train, which are generated mainly by cross-sectional area change of flow channel at the nose and tail of the train (Hara, 1960; Yamamoto, 1973). The cross-sectional area ratio of train to tunnel, or blockage ratio, is a key nondimensional parameter in both phenomena. Also, in the case of long trains, friction on the train surface has a considerable effect. Similarly, the pressure fluctuations also occur in the above-mentioned structures. In particular, when they are constructed as an 'airtight structure' with no openings other than the entrance and exit at both ends, which is the same situation as ordinary tunnels, the pressure fluctuations become large and the problem of insufficient strength of the structure may arise. Moreover, in the case of stations, there can be problems of reduced comfort of passengers and workers on the premises. Therefore, for the purpose of mitigating pressure fluctuations, snow shelters and whole covered stations are often provided with openings (slits, windows, etc.) on their side walls or roof, because they have a remarkable effect to attenuate the pressure waves by emitting them along the whole length of the structure. From a hydrodynamic point of view, these partially enclosed structures are 'vented (leaky) tubes'.

According to the field measurement results on the Shinkansen (Takei et al., 2008; Tokunaga et al., 2015; Haraguchi et al., 2018), the aspect of the pressure fluctuation caused by the train when traveling in these structures is different from that in a tunnel (fully enclosed space) and that in an open air; the pressure fluctuation shows a unique pattern of 'a combination of a single saw tooth and its inverse'. Figure 1 shows an example of measured pressure history on an inner 
side wall surface of the platform floor of a whole covered station equipped with side openings, when a Shinkansen train passes (Takei et. al., 2008). We can see a characteristic pattern of pressure change: gradual rise from 0 (atmospheric) for about $2 \mathrm{~s}$, sudden drop to 0 for about $0.3 \mathrm{~s}$ at the nose passage, maintaining 0 for about $3 \mathrm{~s}$, gradual drop from 0 for about $2 \mathrm{~s}$, and sudden rise to 0 for about $0.3 \mathrm{~s}$ at the tail passage. (The small negative and positive pulses at around $3.2 \mathrm{~s}$ are due to passing of the coupling part of two streamlined trainsets and is not directly related to the subject of this paper.) Similar results were also obtained in experiments conducted at a moving model rig test facility using a 1/25 scale ICE2 train model (Gilbert et. al., 2013). It is evident that the openings such as slits not only attenuate the pressure waves, but also relieve the pressure fluctuations caused by the quasi-steady flow field around the moving train by venting air.

In order to predict this pressure pattern, the authors presented an analysis which combines a one-dimensional steady flow model and a three-dimensional potential flow model around a train running in a long uniform tube with a constant width slit and concluded that the analytical result is in good agreement with the corresponding model experiment result under the condition that the width of the slit, or the opening area ratio, is not large (less than about 3\%) (Iida, et al., 2005). The present paper reports the part of the one-dimensional analysis in this presentation. Although criticism is expected that submission of a paper more than 10 years after the original presentation is untimely, the authors believe that the present one-dimensional analysis is simple but can provide useful knowledge for physical interpretation of the pressure distribution which is peculiar to the partially enclosed structures in high-speed railway.

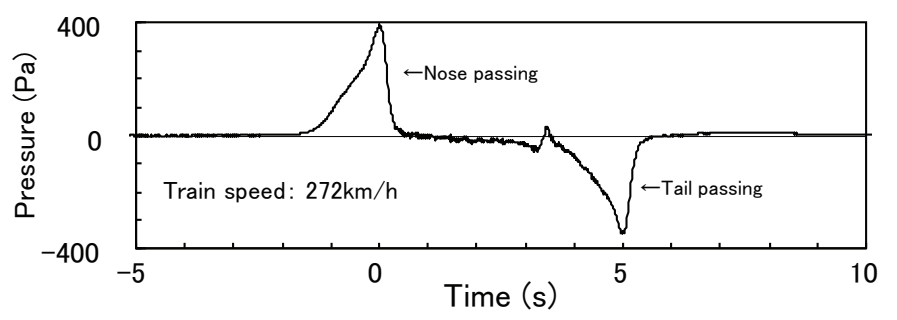

Fig. 1 An example of pressure history on an inner side wall of a whole covered station with side openings at the passage of a high-speed train. Field measrement data on Shinkansen line (Takei, et al., 2008).

\section{One-dimensional flow analysis}

The problem dealt with here is shown in Fig. 2. The tube has a uniform rectangular cross section (width $w$, height $h$ ) and has a slit of a constant width $S_{p}$ running in the longitudinal direction on the tube surface. In principle, the cross section of the tube in the present analysis does not have to be rectangular, and different cross-sectional shapes can be dealt with as long as their area and circumference are known. In the figure, the slit is on the roof, but as far as one-dimensional flow model is concerned, there is no difference when it is on the side wall. The thickness of the wall on which the slit is cut is assumed infinitesimally thin. The shape of the openings of the roof or the side wall of actual snow shelters and whole covered stations is not necessarily slit-like, but may be window-like or porous surface. In these cases, some correction of the analysis is needed for accurate prediction. However, if the openings are bored at a constant rate in the longitudinal direction on average, it can be considered that the situation is equivalent to that shown in Fig. 2 at least qualitatively.

The coordinate system is shown in Fig. 2, where the train runs at a speed of $V$ in the negative direction of the $x_{1}$ axis. The tube is sufficiently long and the pressure waves which are generated by the entering train and propagated through the tube ahead of the train with a speed of sound attenuates within an early period of the train's travel through the tube because the pressure waves continuously radiate from the slit to the outside, which is not the case when the train travels through a tunnel. Therefore, when observed from a frame of reference fixed to the running train, the flow during the train's travel in the tube can be regarded as steady except during the short time period around entry and exit of the train. The train is also long enough that the flow around the nose and tail can be treated separately.

An incompressible fluid is assumed, because the running Mach number $M$ of the train is below 0.4 and the pressure waves can be ignored as described above. The relative change of air density is estimated to be approximately $(1 / 2) M^{2}$ and order of 0.1 when $M=0.4$. The effect of viscosity appears mainly as friction on the surface of the train and the tube inner wall, and also as separation at the tail (leading cars of high-speed trains usually have a streamlined nose shape). In this analysis, the flow separation at the tail is treated in the pressure loss term while the friction on the wall surface of the train and the tube is not directly considered for the following reason. In a pipe flow, the pressure loss due to wall friction 
accumulates downstream, and the pressure gradually drops. However, when there is a considerable leak in the pipe wall, the pressure difference between the inside and outside of the pipe cannot be maintained in a steady flow and, therefore, the gradual pressure drop in the longitudinal direction due to wall friction is hardly observed. Therefore, as far as the purpose is to analyze the one-dimensional pressure field, ignoring wall friction is not considered to have a significant influence. (Of course, when investigating the multidimensional flow velocity distribution or aerodynamic drag of the train, wall friction should be treated appropriately.) However, the wall friction is indirectly considered here in the manner that there is an infinitesimally small but non-zero wall friction in the far upstream region from the train. Then, observed from a frame of reference fixed to the tube, it can be assumed that there is no flow in the region.

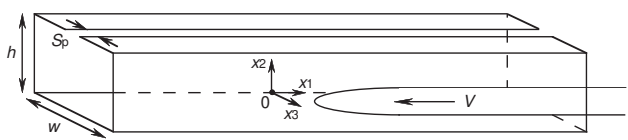

Fig. 2 A train running in a tube with a constant width slit.

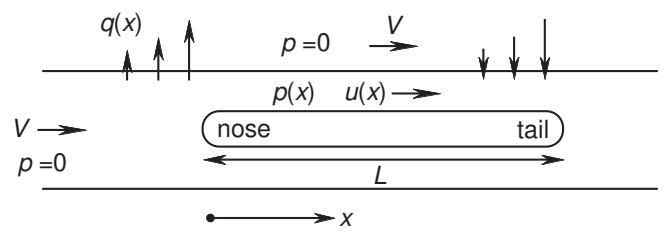

Fig. 3 Analytical model of steady one-dimensional flow observed from a frame of reference fixed to the train.

\subsection{Basic equations of one-dimensional steady flow}

Figure 3 shows an analytical model of one-dimensional steady flow around a train running in a tube with a constant width slit. Observed from a frame of reference fixed to the running train, there is a uniform flow to the right at a velocity of $V$ in the far upstream region inside the tube and also the entire outside region. Pressure is defined as difference from the atmospheric pressure (i. e., gauge pressure), and therefore it is zero in these regions. Hereafter, the $x_{1}$ axis is redefined as the $x$ axis. The origin is set at the position of the nose of the train (for example, at the position where the cross-sectional area of the nose becomes half of that of the uniform part of the train). The pressure inside the tube is $p(x)$, the flow velocity inside the tube is $u(x)$, and the velocity component in the direction perpendicular to the $x$ axis of the flow passing through the slit is $q(x)$, whose sign is positive when the flow is from the inside to the outside.

Applying the mass conservation law to the one-dimensional fluid element of Fig. 4 with the cross section of Fig. 5 gives

$$
\begin{array}{ll}
\rho u A=\rho(u+d u) A+\rho q\left(\sigma S_{p} d x\right), & \text { (where there is no train }), \\
\rho u\left(A-A_{0}\right)=\rho(u+d u)\left(A-A_{0}\right)+\rho q\left(\sigma S_{p} d x\right), & \text { (where there is a train ) }
\end{array}
$$

and therefore

$$
\begin{aligned}
& \frac{d u}{d x}+\beta_{m} q=0, \\
& \beta_{m}= \begin{cases}\frac{\sigma \phi S}{A}, & (m=0, \text { if there is no train }), \\
\frac{\sigma \phi S}{(1-R) A}, & (m=1, \text { if there is a train })\end{cases}
\end{aligned}
$$

where $\rho$ : the density, $w$ : the width of the tube cross section, $h$ : the height of the tube cross section, $A(=w h)$ : the crosssectional area of the tube, $S(=2(w+h))$ : the perimeter of the tube cross section, $S_{p}$ : the width of the slit, $\phi\left(=S S_{p} / S\right)$ : the opening ratio, $A_{0}$ : the cross-sectional area of the train, $R\left(=A_{0} / A\right)$ : the blockage ratio (the cross-sectional area ratio of the train to the tube). $\sigma$ is the coefficient of contraction of jet from the slit, which takes a value in the range of $1 / 2 \sim 1$ (Imai, 1973, p.50).

Applying the momentum conservation law to the fluid element in the section without a train gives

$$
\begin{aligned}
\rho u^{2} A+p A & =\rho(u+d u)^{2} A+(p+d p) A+\rho q u\left(\sigma S_{p} d x\right), \quad(q>0, \text { outflow }), \\
\rho u^{2} A+p A & =\rho(u+d u)^{2} A+(p+d p) A+\rho q V\left(\sigma S_{p} d x\right), \quad(q<0, \text { inflow })
\end{aligned}
$$

Similar equations for the section with a train are obtained by changing the cross-sectional area. Here, it is assumed that the momentum in the $x$ direction is retained while the flow passes through the slit (Steinrück and Sockel, 1985). Therefore, 
in the cases that the width of the slit changes suddenly in the longitudinal direction, that the tube wall is thick, or that the opening is not slit-like (e.g., perforated plate wall), this assumption will result in some modelling error. From the above equations, we have the following differential equation for both cases with and without a train.

$$
\begin{aligned}
& 2 \rho u \frac{d u}{d x}+\beta_{m} \rho q\{K u+(1-K) V\}+\frac{d p}{d x}=0, \\
& K=K(q)= \begin{cases}0, & (q<0), \\
1, & (q>0)\end{cases}
\end{aligned}
$$

Substituting Eq. (1) into Eq. (3) and integrating it gives

$$
p+\frac{1}{2} \rho u^{2}+(1-K) \frac{1}{2} \rho(u-V)^{2}=\text { const. }
$$

where the constant on the right hand side is determined from upstream conditions. Eq. (5) shows that the total pressure of the flow in the tube is constant when $q>0$ but drops due to mixing loss when $q<0$.

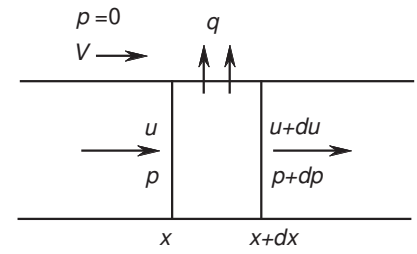

Fig. 4 One-dimensional fluid element.

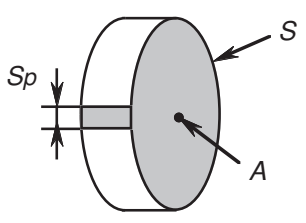

(a) no train

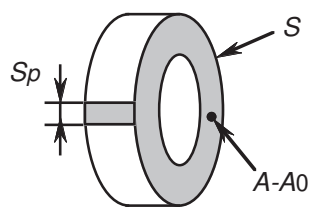

(b) single train

Fig. 5 Simplified model of cross section of a tube with a slit.

\subsection{Velocity of the flow passing through the slit}

The flow through the slit is driven by the pressure difference on both sides of the tube in either case of outflow or inflow. It is assumed that the flow through the slit separates at the edges and forms a jet in the region on the lower pressure side while keeping the velocity in the $x$ direction unchanged as described above. Then, as the Bernoulli equation for steady flow holds along a streamline in the flow passing through the slit, the velocity component in the perpendicular direction $q$ is given by

$$
q=q(p)=\operatorname{sgn}(p) \sqrt{\frac{2|p|}{\rho}}
$$

In the same manner as the momentum equation (3), this assumption about the slit flow will result in the error in calculating $q$ from Eq. (6) when the opening is not slit-like. Regarding this point, Kikuchi et al. performed an analysis using the unsteady Bernoulli equation described in the ground-fixed coordinate system for complicalted shape openings such as windows with an eave, louvers, etc. that are installed in actual Shinkansen stations in order to examine the validity of the simple treatment of Eq. (6) and concluded that pressure loss of complicated openings can be determined by CFD and that the unsteady term in the generalized Bernoulli equation can be neglected if the pressure changes gradually, proposing a dimensionless time constant as an index for criteria (Kikuchi et al., 2010).

\subsection{Jump conditions at discontinuous boundaries of the nose and tail}

Complicated flow fields are formed in the regions near the nose and tail of the train in the tube, and three-dimensional analysis is required to investigate the details of these regions. However, as the present one-dimensional analysis mainly focuses on the region where the flow can be regarded as parallel, corresponding to the gradual pressure changes in the pressure history, we treat the nose and tail of the train as discontinuous boundaries and analyze only the magnitude of change in velocity and pressure across the boundaries from jump conditions based on the conservation laws. Moreover, the slit is not considered in these near field regions, which corresponds with the treatment of the discontinuous boundaries.

Let the total length of the train be $L$, and define symbols as shown in Fig. 6 . There is pressure loss at the tail boundary because of flow separation, while there is not at the nose boundary. Then, from the conservation law of mass and energy, 
the following jump conditions are obtained.

At the nose boundary $(x=0)$ :

$$
\begin{aligned}
& u_{1} A=u_{2}\left(A-A_{0}\right), \\
& p_{1}+\frac{1}{2} \rho u_{1}^{2}=p_{2}+\frac{1}{2} \rho u_{2}^{2}
\end{aligned}
$$

At the tail boundary $(x=L)$ :

$$
\begin{aligned}
& u_{3}\left(A-A_{0}\right)=u_{4} A, \\
& p_{3}+\frac{1}{2} \rho u_{3}^{2}-\frac{1}{2} \rho u_{3}^{2} \xi R^{2}=p_{4}+\frac{1}{2} \rho u_{4}^{2}
\end{aligned}
$$

where $\xi$ is a coefficient related to the pressure loss coefficient of an expansion tube $\zeta$ as $\zeta=\xi R^{2}$, and takes a value in the range of approximately $0 \sim 1$ (JSME, 2006).

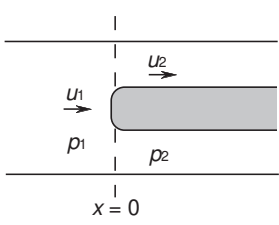

(a) nose boundary

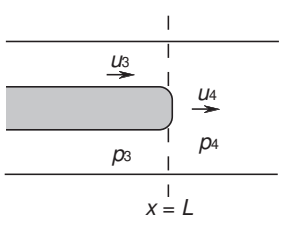

(b) tail boundary
Fig. 6 Discontinuous boundaries at nose and tail

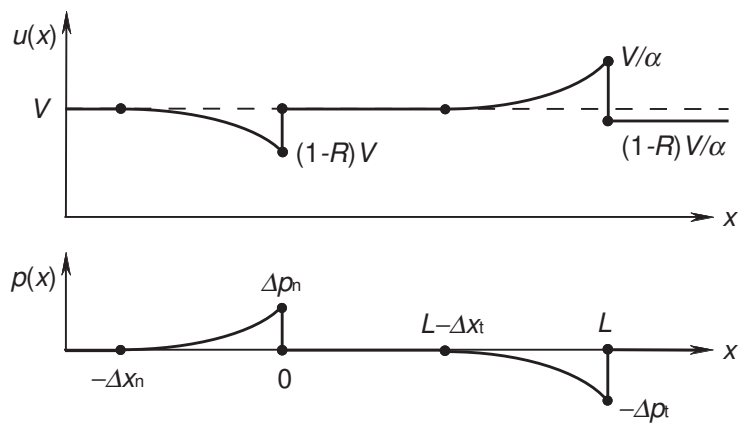

Fig. 7 One-dimensional distribution of velocity and pressure around a train in a tube with a constant width slit.

\subsection{Analytical results}

Equations (1), (3), (6) implies that $q>0, d u / d x<0, d p / d x>0$ in the region where $p>0$, and that $q<0, d u / d x>0$, $d p / d x<0$ in the region where $p<0$. Moreover, the relation $p_{1}>p_{2}$ holds from Eqs. (7), (8), and $p_{3}<p_{4}$ holds from Eqs. (9), (10), respectively. In addition, the condition of $p=0$ is satisfied in the region far upstream of the nose, the region downstream of the nose and far upstream of the tail, and the region downstream of the tail.

In the region of $x<0, q \geq 0$ and $p \geq 0$ hold, so that from Eqs. (1),(4),(5),(6) we can obtain the following differential equation with $u(x)$ as an unknown function.

$$
\frac{d u}{d x}+\beta_{0} \sqrt{V^{2}-u^{2}}=0
$$

Here, when using Eq.(5), the constant on the right hand side is set to $\frac{1}{2} \rho V^{2}$ from the upstream condition of $u=V$ and $p=0$. The general solution of Eq. (11) is given by the following trigonometric function.

$$
u(x)=V \sin \left(-\beta_{0} x+C_{n}\right),
$$

where $C_{n}$ is an integral constant. Therefore, from the jump conditions (7), (8) at the nose boundary and the upstream/downstream conditions, the flow velocity and pressure distribution in the regions ahead of and just behind the nose becomes as follows.

$$
\begin{aligned}
& u(x)= \begin{cases}V, & \left(x \leq-\Delta x_{n}, 0 \leq x\right), \\
V \sin \left(-\beta_{0} x+C_{n}\right), & \left(-\Delta x_{n}<x<0\right),\end{cases} \\
& p(x)= \begin{cases}0, & \left(x \leq-\Delta x_{n}, 0 \leq x\right), \\
\frac{1}{4} \rho V^{2}\left\{1+\cos 2\left(-\beta_{0} x+C_{n}\right)\right\},\left(-\Delta x_{n}<x<0\right),\end{cases} \\
& u_{1}=(1-R) V, u_{2}=V, \\
& p_{1}=\Delta p_{n}, p_{2}=0, \Delta p_{n}=\frac{1}{2} \rho V^{2} R(2-R), \\
& \Delta x_{n}=\frac{\cos ^{-1}(1-R)}{\beta_{0}}, C_{n}=\sin ^{-1}(1-R),
\end{aligned}
$$


where $\Delta x_{n}$ is determined by equating the second line of Eq. (13) with $V$.

Similarly, in the region of $0<x<L, q \leq 0$ and $p \leq 0$ hold, so that from Eqs. (1),(4),(5),(6) we obtain the following differential equation.

$$
\frac{d u}{d x}-\sqrt{2} \beta_{1} \sqrt{u^{2}-V u}=0,
$$

Here, when using Eq.(5), the constant on the right hand side is also set to $\frac{1}{2} \rho V^{2}$ from the condition just behind the nose, of $u=V$ and $p=0$. The general solution of Eq. (18) is given by the following hyperbolic function.

$$
u(x)=\frac{V}{2}\left\{\cosh \left(\sqrt{2} \beta_{1} x+C_{t}\right)+1\right\},
$$

where $C_{t}$ is an integral constant. Therefore, from the jump conditions (9), (10) at the tail boundary and the upstream/downstream conditions, the flow velocity and pressure distribution in the regions ahead of and just behind the tail becomes as follows.

$$
\begin{aligned}
& u(x)= \begin{cases}V, & \left(x \leq L-\Delta x_{t}\right), \\
\frac{V}{2}\left\{\cosh \left(\sqrt{2} \beta_{1} x+C_{t}\right)+1\right\}, & \left(L-\Delta x_{t}<x<L\right), \\
(1-R) V / \alpha, & (L \leq x),\end{cases} \\
& p(x)= \begin{cases}0, & \left(x \leq L-\Delta x_{t}, L \leq x\right), \\
-\frac{1}{8} \rho V^{2}\left\{\cosh 2\left(\sqrt{2} \beta_{1} x+C_{t}\right)-1\right\},\left(L-\Delta x_{t}<x<L\right),\end{cases} \\
& u_{3}=\frac{V}{\alpha}, u_{4}=\frac{(1-R) V}{\alpha}, \\
& p_{3}=-\Delta p_{t}, p_{4}=0, \Delta p_{t}=\rho V^{2} \frac{1-\alpha}{\alpha^{2}}, \\
& \Delta x_{t}=\frac{1}{\sqrt{2} \beta_{1}} \cosh ^{-1}\left(\frac{2-\alpha}{\alpha}\right), C_{t}=\cosh ^{-1}\left(\frac{2-\alpha}{\alpha}\right)-\sqrt{2} \beta_{1} L, \\
& \alpha=1-R+\frac{1+\xi}{2} R^{2}
\end{aligned}
$$

where $\Delta x_{t}$ is determined by equating the second line of Eq. (20) with $V$.

These analytical results are schematically shown in Fig. 7. The overall shape of the distribution of $q(x)$ resembles that of $p(x)$ because of the relation of Eq. (6). The spatial pressure waveform in Fig. 7 can be read as a similar time waveform using the relation of $x=V t$. It can be seen that the characteristic pattern of 'a combination of a single saw tooth and its inverse' of the measured waveform in Fig. 1 has been reproduced in the figure.

\subsection{Comparison with model experiment}

Figure 8 shows a comparsion of the results of the one-dimensional analysis and model experiment for the two cases of the slit opening ratio $\phi=0.01$ and 0.03 . In the figure, the analytical results are shown as a time waveform observed at a fixed point in the tube. In the model experiment, an axisymmetric model train travels in a tube and pressure history inside the tube is measured (Iida et al., 2005). The experimental conditions are shown in Table 1. The results of the one-dimensional analysis in the figure are obtained under the conditions when the coefficient of contraction $\sigma=0.75$ and the coefficient related to pressure loss at the tail $\xi=0.5$.

According to the classical free streamline theory, $\sigma$ for the two-dimensional slit is 0.611 (Imai, 1973, p.239), but in this comparison, setting $\sigma=0.75$ has been found to improve the agreement with the experiment. In order to determine the correct value of $\sigma$, it will be necessary to investigate the flow through the slit in detail. Also it is not easy to determine the proper value of $\xi$ because it depends on the complicated separated flow around the tail. However, as can be seen from Eq. (25), the error in its value does not have a significant influence on the magnitude of pressure when $R$ is small (usually, $R<0.2$ in high-speed railway).

Despite these assumptions, overall patterns of pressure fluctuation in the results of analysis and experiment are similar in both cases of $\phi=0.01$ and 0.03 . In particular, in terms of the size (length) of the regions of positive pressure ahead of the nose and of negative pressure ahead of the tail, the analytical and experimental results are in good agreement, partly due to the adjustment of $\sigma$. On the other hand, regarding the magnitude of pressure drop by the nose passing and the pressure rise by the tail passing, the analytical results exceed the experimental ones, and the difference increases as $\phi$ increases. The main cause of this overestimation is that the nose and tail boundaries are treated as discontinuities and that the effect of the slit is not taken into consideration in the jump conditions as is shown in Eqs. (16) and (23). However, it is considered that this degree of overestimation of pressure loads is rarely a decisive problem in the desigin of large scale structures which are also exposed to various natural external forces such as wind and snow loads. 
Table 1 Conditions of model experiment

\begin{tabular}{|c|c|}
\hline model scale & $1 / 90$ \\
\hline tube height $h(\mathrm{~m})$ & 0.1 \\
\hline$w / h$ & 1.89 \\
\hline blockage ratio $R$ & 0.083 \\
\hline opening ratio $\phi$ & $0.01,0.03$ \\
\hline vertical positon of pressure sensors on inner side wall & at the height of $0.34 h$ from tube floor \\
\hline$V$ (km/h) & 360 \\
\hline$L / h$ & 12.4 \\
\hline nose (tail) length & $0.67 h$ \\
\hline nose (tail) shape & paraboloid of rev. \\
\hline vertical position of train axis & at the height of $0.34 h$ from tube floor \\
\hline horizontal position of train axis & at the center of tube cross-section width \\
\hline
\end{tabular}

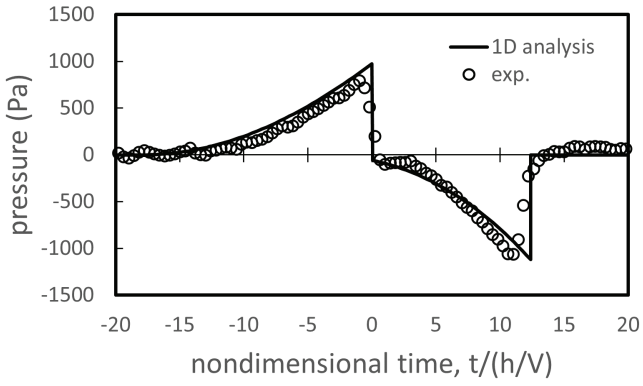

(a) $\phi=0.01$

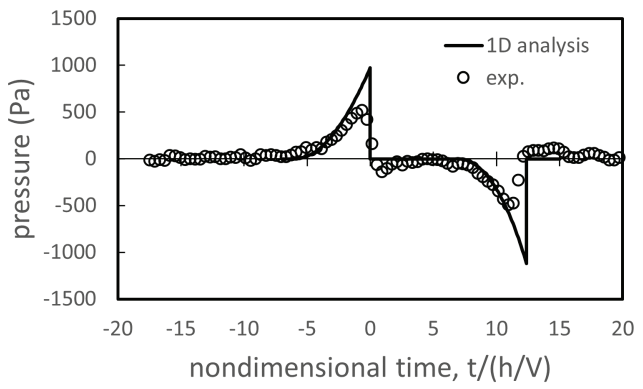

(b) $\phi=0.03$

Fig. 8 Comparison between one-dimensional analysis and model experiment, $V=360 \mathrm{~km} / \mathrm{h}$. In the analysis, $\sigma=0.75$ and $\xi=0.5$.

\section{Discussion}

It can be seen from the flow velocity of Eq. (15) and Fig. 7 that the relative velocity ahead of the nose is lower than the running speed. This is because the air upstream of the nose in the tube flows out to the outside through the slit. On the other hand, in the region upstream of the tail, the air outside the tube flows in through the slit, so that the relative velocity ahead of the tail exceeds the running speed as shown in Eq. (22). The present analysis ignores wall friction and, therefore, quantitative evaluation of air resistance of the train is impossible. However, the result of the flow acceleration upstream of the tail leads us to a qualitative, yet important finding that there exists a unique mechanism for increase of air resistance of a train running in partially enclosed structures.

Furthermore, it can be seen from Fig. 7 that the flow velocity downstream of the tail does not recover to $V$, and that, observed from a frame of reference fixed to the tube, the air behind the tail is being dragged by the train, which is caused by the formation of a jet at the slit and the wake generation by flow separation at the tail. In Fig. 7, this dragged flow is shown to exist to the downstream infinitely. This is the result of ignoring friction on the tube wall in the analysis, but in reality it recovers to $V$ at a certain point downstream, with air supplied from the outside through the slit.

From Eqs. (17) and (24), the region of positive pressure ahead of the nose and that of negative pressure ahead of the tail are limited in the $x$ direction. Their sizes $\Delta x_{n}$ and $\Delta x_{t}$ are directly related to the degree of airtightness of the tube (determined by the opening ratio $\phi$ and the coefficient of contraction $\sigma$ through $\beta_{m}$ defined in Eq. (2)) and does not depend on the running speed. Using these equations, it may become possible to estimate the airtightness of the structures through pressure measurement in running test.

Equations (16) and (23) are the magnitudes of the pressure drop by the nose passing and the pressure rise by the tail passing, respectively and implies that $\Delta p_{n}<\Delta p_{t}$ regardless of the value of $\xi(>0)$. It is known that, when a train having the same shape for its nose and tail passes by in an open air section, the pressure change by tail passing is smaller than that by nose passing due to the thick boundary layer along a long train and the flow separation at the tail (e.g., Kikuchi et al., 1996). In the tunnel section, the magnitude of pressure rise at the tail is also usually smaller than that of pressure drop at the nose because of pressure loss caused by separation at the tail. However, the present result of the tube with a slit is the opposite of these, which appears to be strange at first glance, but the cause is clear. As described above, the 
flow velocity ahead of the nose decelerates from $V$ and the velocity ahead of the tail accelerates from $V$ as a result of flow through the slit and this affects directly the pressure change at the nose and tail. This point is remarkably different from the tunnel case where the flow rate is conserved only in the tube.

The actual pressure fields near the nose and tail are continuous and three-dimensional, not discontinuous boundaries as in the one-dimensional model. The specific form of the pressure change at train passing depends on train shape and three-dimensional positional relationship between the train and the pressure evaluation point in a tube or tunnel. Consequently, measurement data of pressure fluctuations in a shelter or whole covered station does not necessarily accord with the conclusion of one-dimensional analysis: $\Delta p_{n}<\Delta p_{t}$. For example, the pressure drop at the nose is slightly larger than the pressure rise at the tail in the field measurement result shown in Fig. 1. On the other hand, the pressure drop at the nose is smaller than the pressure rise at the tail in the model experiment result shown in Fig. 8(a). To summarize, when the nose and tail have the same shape the pressure change at the nose always exceeds that at the tail in an open air or tunnel, but in a partially enclosed structure, which of the pressure change at the nose or at the tail is larger depends on the conditions.

The present one-dimensional analysis provides basic knowledge for understanding the steady pressure field around the train running in a partially enclosed structure, and leads to simple formulae allowing rough estimation of the onedimensional pressure load on the structure. However, if it is required to predict more realistic and detailed pressure distribution, we need to conduct three-dimensional analysis, such as potential flow analysis and CFD. Even in that case, by combining the one-dimensional analysis with three-dimensional analysis, the efficiency of the overall computation can be significantly improved. One straightforward method of such combined analysis is to use the one-dimensional formlae as boundary conditions of the three-dimensional computational domains of the near field flow around the nose and tail.

\section{References}

Gilbert, T., Baker, C. and Quinn, A., Aerodynamic pressures around high-speed trains: the transition from unconfined to enclosed spaces, Proceedings of the Institution of Mechanical Engineers, Part F: Journal of Rail and Rapid Transit, Vol. 227 (2013), pp. 609-622.

Hara, T., Aerodynamic force acting on a high speed train at the tunnel entrance, Transactions of the Japan Society of Mechanical Engineers, Vol.26, No.171 (1960), pp.1581-1586 (in Japanese).

Haraguchi, K., Sato, J., Hayashi, A., Takei, Y. and Izumi, Y., A study on evaluation of pressure variation in station having all covering roof under high-speed train passage, Journal of Environmental Engineering, Architectural Institute of Japan, Vol.83, No.744 (2018), pp.159-169 (in Japanese).

Iida, M., Kikuchi, K., Takei, Y. and Izumi, Y., Steady pressure field around a train running in a vented tube, Proceedings of JSME Fluid Engineering Conference 2005, CD-ROM, No.1207 (2005) (in Japanese).

Imai, I., Fluid Dynamics, Vol. I. (1973), Syokabo (in Japanese).

JSME ed., JSME Mechanical Engineers' Handbook, Fundamentals, $\alpha$ 4: Fluid Engineering, The Japan Society of Mechanical Engineers (2006), p.72 (in Japanese).

Kikuchi, K., Uchida, K., Nakatani, K., Yoshida, Y., Maeda, T. and Yanagizawa, M., Numerical analysis of pressure variation due to train passage using the boundary element method, Quarterly Report of Railway Techinical Research Institute, Vol.37, No.4 (1996), pp.231-237.

Kikuchi, K., Ozawa, S., Takei, Y., Yoshida, Y. and Kajiyama, H., A study on a pressure condition under which a flow discharged from an opening of a whole covering shed of a railway station can be regarded as a quasi-steady flow, Transactions of the Japan Society of Mechanical Engineers, Series B, Vol.76, No.766 (2010), pp.933-942 (in Japanese).

Steinrück P. and Sockel, H., Further calculations on transient pressure alleviation and simplified formulae for initial tunnel design, Proceedings of the 5th International Symposium on the Aerodynamics \& Ventilation of Vehicle Tunnels (1985), Lille, France: 20-22 May, 1985, pp.317-341.

Takei, Y., Izumi, Y., Yamada, S., Iida, M. and Kikuchi, K., Evaluation method for air pressure variation and station facility member deterioration caused by high-speed train passage in stations, Quarterly Report of Railway Techinical Research Institute, Vol.49, No.2 (2008), pp.89-95.

Tokunaga, M., Matsuoka, K., Inoue, S., Yamada, T. and Sogabe, M., Dynamic behaviour of steel snow shelters under train passing, Journal of Railway Engineering, Japan Society of Civil Engineers, No.19 (2015), pp.51-58 (in Japanese).

Yamamoto, A., Pressure variations, aerodynamic drag and tunnel ventilation in Shin-kan-sen type tunnel, Railway Technical Research Report, Japanese National Railways, No.871 (1973) (in Japanese). 